\title{
The Role of Phonons in a Topological Material
}

\author{
Unusual interactions occur between phonons and electrons in the \\ topological semimetal tungsten diphosphide, a finding that could explain \\ some of the material's strange properties.
}

By Sophia Chen

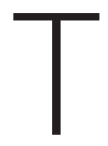

he fundamental interactions underlying many unusual electronic properties of topological semimetals are still not well understood. Now, an investigation by Kenneth Burch of Boston College, Massachusetts, and colleagues into the topological semimetal tungsten diphosphide $\left(\mathrm{WP}_{2}\right)$ has found that the material's peculiar charge-transport behavior is largely due to electron-phonon scattering [1]. The researchers say that understanding the importance of such interactions could guide efforts to discover other similar materials.

Compared to conventional metals, topological semimetals like $W_{2}$ are notable for their extremely high electrical conductance, despite having few free electrons. Similarly perplexing, when the temperature of $\mathrm{WP}_{2}$ rises above about $50 \mathrm{~K}$, this electrical conductance decreases by orders of magnitude. To investigate these effects, Burch and colleagues used Raman spectroscopy to observe the properties of $\mathrm{WP}_{2}$ 's vibrational modes-phonons-at temperatures ranging from 7 to $300 \mathrm{~K}$.

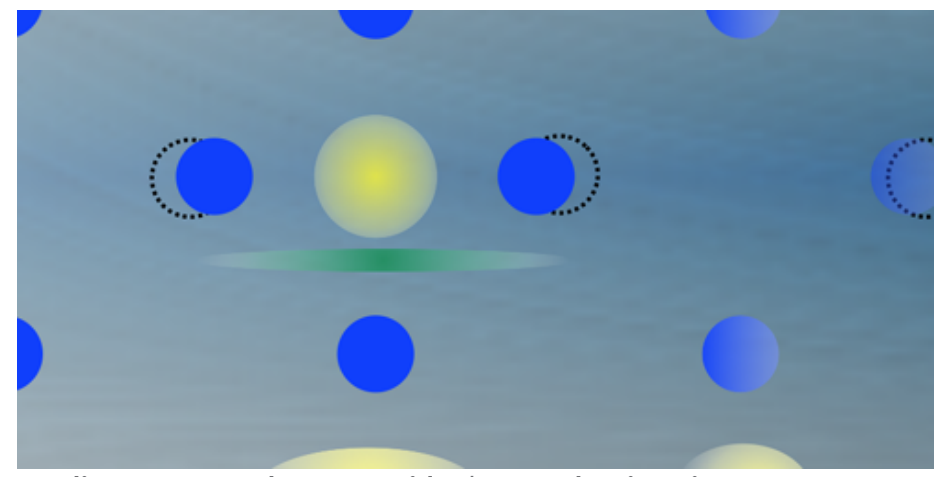

Credit: Y. Wang and G. Varnavides/Harvard University
Then, using first-principles calculations, they studied how these phonons decayed.

Their model of the phonons' energies and momenta suggested that phonons in $\mathrm{WP}_{2}$ tend to decay into electron-hole pairs rather than into other phonons. They also found that at temperatures below $50 \mathrm{~K}$, some phonons decay by scattering off electrons rather than-as in conventional materials-by scattering off other phonons. As a result of this phonon-electron scattering, in $\mathrm{WP}_{2}$, electrons and phonons become coupled, forming quasiparticles that carry electrical current efficiently through the material. This electron mobility gives $W_{2} P_{2}$ its anomalously high conductance at low temperatures.

The researchers plan to look for such phonon-electron scattering in other heavy-atom-containing semimetals. Their ultimate goal is to engineer this effect in materials at higher temperatures.

Sophia Chen is a freelance science writer based in Columbus, Ohio.

\section{REFERENCES}

1. G. Osterhoudt et al., "Evidence for dominant phonon-electron scattering in Weyl semimetal WP 2 ," Phys. Rev. X 11, 011017 (2021). 